Groups Geom. Dyn. 7 (2013), 535-541

DOI $10.4171 / \mathrm{GGD} / 195$
Groups, Geometry, and Dynamics

(C) European Mathematical Society

\title{
Regular elements in CAT(0) groups
}

\author{
Pierre-Emmanuel Caprace ${ }^{1}$ and Gašper Zadnik ${ }^{2}$
}

\begin{abstract}
Let $X$ be a locally compact geodesically complete CAT( 0$)$ space and $\Gamma$ be a discrete group acting properly and cocompactly on $X$. We show that $\Gamma$ contains an element acting as a hyperbolic isometry on each indecomposable de Rham factor of $X$. It follows that if $X$ is a product of $d$ factors, then $\Gamma$ contains $\mathbb{Z}^{d}$.
\end{abstract}

Mathematics Subject Classification (2010). 20 F65.

Keywords. CAT(0) group, regular elements, flat closing conjecture.

Let $X$ be a proper CAT(0) space and $\Gamma$ be a discrete group acting properly and cocompactly by isometries on $X$. The flat closing conjecture predicts that if $X$ contains a $d$-dimensional flat, then $\Gamma$ contains a copy of $\mathbb{Z}^{d}$ (see [Gro93], Section 6.B 3 ). In the special case $d=2$, this would imply that $\Gamma$ is hyperbolic if and only if it does not contain a copy of $\mathbb{Z}^{2}$. This notorious conjecture remains however open as of today. It holds when $X$ is a real analytic manifold of non-positive sectional curvature by the main result of [BS91]. In the classical case when $X$ is a non-positively curved symmetric space, it can be established with the following simpler and well known argument: by [BL93], Appendix, the group $\Gamma$ must contain a so called $\mathbb{R}$-regular semisimple element, i.e., a hyperbolic isometry $\gamma$ whose axes are contained in a unique maximal flat of $X$. By a lemma of Selberg [Sel60], the centraliser $\mathcal{Z}_{\Gamma}(\gamma)$ is a lattice in the centraliser $\mathcal{Z}_{\text {Isom }(X)}(\gamma)$. Since the latter centraliser is virtually $\mathbb{R}^{d}$ with $d=\operatorname{rank}(X)$, one concludes that $\Gamma$ contains $\mathbb{Z}^{d}$, as desired.

It is tempting to try and mimic that strategy of proof in the case of a general CAT(0) space $X$ : if one shows that $\Gamma$ contains a hyperbolic isometry $\gamma$ which is maximally regular in the sense that its axes are contained in a unique flat of maximal possible dimension among all flats of $X$, then the flat closing conjecture will follow as above. The main result of this note provides hyperbolic isometries satisfying a weaker notion of regularity.

\footnotetext{
${ }^{1}$ F.R.S.-FNRS Research Associate, supported in part by FNRS (grant F.4520.11) and the European Research Council (grant \#278469).

${ }^{2}$ Supported by the Slovenian Research Agency and in part by the Slovene Human Resources Development and Scholarship Fund.
} 
Theorem. Assume that $X$ is geodesically complete.

Then $\Gamma$ contains a hyperbolic element which acts as a hyperbolic isometry on each indecomposable de Rham factor of $X$.

Every CAT( 0$)$ space $X$ as in the theorem admits a canonical de Rham decomposition, see [CM09a], Corollary 5.3 (ii). Notice that the number of indecomposable de Rham factors of $X$ is a lower bound on the dimension of all maximal flats in $X$, although two such maximal flats need not have the same dimension in general. As expected, we deduce a corresponding lower bound on the maximal rank of free abelian subgroups of $\Gamma$.

Corollary 1. If $X$ is a product of $d$ factors, then $\Gamma$ contains a copy of $\mathbb{Z}^{d}$.

We believe that those results should hold without the assumption of geodesic completeness; in case $X$ is a CAT(0) cube complex, this is indeed so, see [CS11], $\S 1.3$.

The proof of the theorem and its corollary relies in an essential way on results from [CM09a] and [CM09b]. The first step consists in applying [CM09a], Theorem 1.1, which ensures that $X$ splits as

$$
X \cong \mathbb{R}^{d} \times M \times Y_{1} \times \cdots \times Y_{q},
$$

where $M$ is a symmetric space of non-compact type and the factors $Y_{i}$ are geodesically complete indecomposable $\mathrm{CAT}(0)$ spaces whose full isometry group is totally disconnected. Moreover this decomposition is canonical, hence preserved by a finite index subgroup of $\operatorname{Isom}(X)$ (and thus of $\Gamma$ ). The next essential point is that, by [CM09b], Theorem 3.8, the group $\Gamma$ virtually splits as $\mathbb{Z}^{d} \times \Gamma^{\prime}$, and the factor $\Gamma^{\prime}$ (resp. $\mathbb{Z}^{d}$ ) acts properly and cocompactly on $M \times Y_{1} \times \cdots \times Y_{q}$ (resp. $\mathbb{R}^{d}$ ). Therefore, our main theorem is a consequence of the following.

Proposition 2. Let $X=M \times Y_{1} \times \cdots \times Y_{q}$, where $M$ is a symmetric space of non-compact type and $Y_{i}$ is a geodesically complete locally compact $\mathrm{CAT}(0)$ space with totally disconnected isometry group.

Any discrete cocompact group of isometries of $X$ contains an element acting as an $\mathbb{R}$-regular hyperbolic element on $M$, and as a hyperbolic element on $Y_{i}$ for all $i$.

As before, this yields a lower bound on the rank of maximal free abelian subgroups of $\Gamma$, from which Corollary 1 follows.

Corollary 3. Let $X=M \times Y_{1} \times \cdots \times Y_{q}$ be as in the proposition. Then any discrete cocompact group of isometries of $X$ contains a copy of $\mathbb{Z}^{\operatorname{rank}(M)+q}$.

Proof. Let $\Gamma<\operatorname{Isom}(X)$ be a discrete subgroup acting cocompactly. Upon replacing $\Gamma$ by a subgroup of finite index, we may assume that $\Gamma$ preserves the given product 
decomposition of $X$ (see [CM09a], Corollary 5.3(ii)). Let $\gamma \in \Gamma$ be as in Proposition 2 and let $\gamma_{M}$ (resp. $\gamma_{i}$ ) be its projection to $\operatorname{Isom}(M)\left(\operatorname{resp}\right.$. Isom $\left.\left(Y_{i}\right)\right)$. Then $\operatorname{Min}\left(\gamma_{M}\right)=\mathbb{R}^{\operatorname{rank}(M)}$ and for all $i$ we have $\operatorname{Min}\left(\gamma_{i}\right) \cong \mathbb{R} \times C_{i}$ for some $\operatorname{CAT}(0)$ space $C_{i}$, by [BH99], Theorem II.6.8 (5). Hence the desired conclusion follows from the following lemma.

Lemma 4. Let $X=X_{1} \times \cdots \times X_{p}$ be a proper $\mathrm{CAT}(0)$ space and $\Gamma$ a discrete group acting properly cocompactly on $X$. Let also $\gamma \in \Gamma$ be an element preserving some $d_{i}$-dimensional flat in $X_{i}$ on which it acts by translation, for all $i$.

Then $\Gamma$ contains a free abelian group of rank $d_{1}+\cdots+d_{p}$.

Proof. By assumption $\gamma$ preserves the given product decomposition of $X$. We let $\gamma_{i}$ denote the projection of $\gamma$ on $\operatorname{Isom}\left(X_{i}\right)$. Observe that

$$
\operatorname{Min}(\gamma)=\operatorname{Min}\left(\gamma_{1}\right) \times \cdots \times \operatorname{Min}\left(\gamma_{p}\right) .
$$

By hypothesis, we have $\operatorname{Min}\left(\gamma_{i}\right) \cong \mathbb{R}^{d_{i}} \times C_{i}$ for some CAT(0) space $C_{i}$. Therefore $\operatorname{Min}(\gamma) \cong \mathbb{R}^{d_{1}+\cdots+d_{p}} \times C_{1} \times \cdots \times C_{p}$. By [Rua01], Theorem 3.2, the centraliser $\mathcal{Z}_{\Gamma}(\gamma)$ acts cocompactly (and of course properly) on $\operatorname{Min}(\gamma)$. Therefore, in view of [CM09b], Theorem 3.8, we infer that $Z_{\Gamma}(\gamma)$ contains a subgroup isomorphic to $\mathbb{Z}^{d_{1}+\cdots+d_{p}}$.

It remains to prove Proposition 2. We proceed in three steps. The first one provides an element $\gamma_{Y} \in \Gamma$ acting as a hyperbolic isometry on each $Y_{i}$. This combines an argument of E. Swenson [Swe99], Theorem 11, with the phenomenon of Alexandrov angle rigidity, described in [CM09a], Proposition 6.8, and recalled below. The latter requires the hypothesis of geodesic completeness. The second step uses that $\Gamma$ has subgroups acting properly cocompactly on $M$, and thus contains an element $\gamma_{M}$ acting as an $\mathbb{R}$-regular isometry of $M$ by [BL93]. The last step uses a result from [PR72] ensuring that for all elements $\delta^{\prime}$ in some Zariski open subset of Isom( $\left.M\right)$ and all sufficiently large $n>0$, the product $\gamma_{M}^{n} \delta^{\prime}$ is $\mathbb{R}$-regular. Invoking the Borel density theorem, we finally find an appropriate element $\delta \in \Gamma$ such that the product $\gamma=\gamma_{M}^{n} \delta \gamma_{Y}$ has the requested properties. We now proceed to the details.

Proposition (Alexandrov angle rigidity). Let $Y$ be a locally compact geodesically complete $\mathrm{CAT}(0)$ space and $G$ be a totally disconnected locally compact group acting continuously, properly and cocompactly on $Y$ by isometries.

Then there is $\varepsilon>0$ such that for any elliptic isometry $g \in G$ and any $x \in X$ not fixed by $g$, we have $L_{c}(g x, x) \geq \varepsilon$, where $c$ denotes the projection of $x$ on the set of $g$-fixed points.

Proof. See [CM09a], Proposition 6.8. 
Proposition 5. Let $Y=Y_{1} \times \cdots \times Y_{q}$, where $Y_{i}$ is a geodesically complete locally compact $\mathrm{CAT}(0)$ space with totally disconnected isometry group, and $G$ be a locally compact group acting continuously, properly and cocompactly by isometries on $Y$.

Then $G$ contains an element acting on $Y_{i}$ as a hyperbolic isometry for all $i$.

Proof. Upon replacing $G$ by a finite index subgroup, we may assume that $G$ preserves the given product decomposition of $Y$, see [CM09a], Corollary 5.3 (ii). Let $\rho:[0, \infty) \rightarrow Y$ be a geodesic ray which is regular, in the sense that its projection to each $Y_{i}$ is a ray (in other words the end point $\rho(\infty)$ does not belong to the boundary of a subproduct).

Since $G$ is cocompact, we can find a sequence $\left(g_{n}\right)$ in $G$ and a strictly increasing sequence $\left(t_{n}\right)$ in $\mathbb{Z}_{+}$such that the sequence of maps

$$
\rho_{n}:\left[-t_{n}, \infty\right) \rightarrow Y, \quad t \mapsto g_{n} \cdot \rho\left(t+t_{n}\right),
$$

converges uniformly on compact subsets of $\mathbb{R}$ to a geodesic line $\ell: \mathbb{R} \rightarrow Y$. Set $h_{i, j}=g_{i}^{-1} g_{j} \in G$ and consider the angle

$$
\theta=\angle_{\rho\left(t_{i}\right)}\left(h_{i, j}^{-1} \cdot \rho\left(t_{i}\right), h_{i, j} \cdot \rho\left(t_{i}\right)\right) .
$$

As in [Swe99], Theorem 11, observe that $\theta$ is arbitrarily close to $\pi$ for $i<j$ large enough.

We shall prove that for all $i<j$ large enough, the isometry $h_{i, j}$ is regular hyperbolic, in the sense that its projection to each factor $Y_{k}$ is hyperbolic. We argue by contradiction and assume that this is not the case. Notice that $\operatorname{Isom}\left(Y_{k}\right)$ does not contain any parabolic isometry by [CM09a], Corollary 6.3 (iii). Therefore, upon extracting and reordering the factors, we may then assume that there is some $s \leq q$ such that for all $i<j$, the projection of $h_{i, j}$ on $\operatorname{Isom}\left(Y_{1}\right), \ldots, \operatorname{Isom}\left(Y_{S}\right)$ is elliptic, and the projection of $h_{i, j}$ on $\operatorname{Isom}\left(Y_{s+1}\right), \ldots$, Isom $\left(Y_{q}\right)$ is hyperbolic. We set $Y^{\prime}=$ $Y_{1} \times \cdots \times Y_{s}$ and $Y^{\prime \prime}=Y_{s+1} \times \cdots \times Y_{q}$. We shall prove that for $i<j$ large enough, the projections of $\left(h_{i, j}\right)$ on Isom $\left(Y^{\prime}\right)$ forms a sequence of elliptic isometries which contradict Alexandrov angle rigidity.

Fix some small $\delta>0$. Let $x_{i}$ (resp. $y_{i}$ ) be the point at distance $\delta$ from $\rho\left(t_{i}\right)$ and lying on the geodesic segment $\left[h_{i, j}^{-1} \cdot \rho\left(t_{i}\right), \rho\left(t_{i}\right)\right]$ (resp. $\left.\left[\rho\left(t_{i}\right), h_{i, j} . \rho\left(t_{i}\right)\right]\right)$. By construction, for $i<j$ large enough, the union of the two geodesic segments $\left[x_{i}, \rho\left(t_{i}\right)\right] \cup\left[\rho\left(t_{i}\right), y_{i}\right]$ lies in an arbitrary small tubular neighbourhood of the geodesic ray $\rho$. Since the projection $Y \rightarrow Y^{\prime}$ is 1-Lipschitz, it follows that the $Y^{\prime}$-component of $\left[x_{i}, \rho\left(t_{i}\right)\right] \cup\left[\rho\left(t_{i}\right), y_{i}\right]$, which we denote by $\left[x_{i}^{\prime}, \rho^{\prime}\left(t_{i}\right)\right] \cup\left[\rho^{\prime}\left(t_{i}\right), y_{i}^{\prime}\right]$, is uniformly close to the $Y^{\prime}$-component of $\rho$, say $\rho^{\prime}$. Since $\rho$ is a regular ray, its projection $\rho^{\prime}$ is also a geodesic ray. Therefore, the angle

$$
\theta^{\prime}=\angle_{\rho^{\prime}\left(t_{i}\right)}\left(x_{i}^{\prime}, y_{i}^{\prime}\right)
$$

is arbitrarily close to $\pi$ for $i<j$ large enough. Pick $i<j$ so large that $\theta^{\prime}>\pi-\varepsilon$, where $\varepsilon>0$ is the constant from Alexandrov angle rigidity for $Y^{\prime}$. Set $h=h_{i, j}$ and 
let $h^{\prime}$ be the projection of $h$ on $\operatorname{Isom}\left(Y^{\prime}\right)$. By assumption $h^{\prime}$ is elliptic. Let $c$ denote the projection of $\rho^{\prime}\left(t_{i}\right)$ on the set of $h^{\prime}$-fixed points. Then the isosceles triangles $\triangle\left(c,\left(h^{\prime}\right)^{-1} \cdot \rho^{\prime}\left(t_{i}\right), \rho^{\prime}\left(t_{i}\right)\right)$ and $\triangle\left(c, \rho^{\prime}\left(t_{i}\right), h^{\prime} \cdot \rho^{\prime}\left(t_{i}\right)\right)$ are congruent, and we deduce

$$
\begin{aligned}
\angle_{c}\left(\rho^{\prime}\left(t_{i}\right), h^{\prime} \cdot \rho^{\prime}\left(t_{i}\right)\right) & \leq \pi-\angle_{\rho^{\prime}\left(t_{i}\right)}\left(c, h^{\prime} \cdot \rho^{\prime}\left(t_{i}\right)\right)-\angle_{\rho^{\prime}\left(t_{i}\right)}\left(c,\left(h^{\prime}\right)^{-1} \cdot \rho^{\prime}\left(t_{i}\right)\right) \\
& \leq \pi-\angle_{\rho^{\prime}\left(t_{i}\right)}\left(\left(h^{\prime}\right)^{-1} \cdot \rho^{\prime}\left(t_{i}\right), h^{\prime} \cdot \rho^{\prime}\left(t_{i}\right)\right) \\
& =\pi-\theta^{\prime} \\
& <\varepsilon .
\end{aligned}
$$

This contradicts Alexandrov angle rigidity.

Proof of Proposition 2. Let $\Gamma$ be a discrete group acting properly and cocompactly on $X$. First observe that (after passing to a finite index subgroup) we may assume that $\Gamma$ preserves the given product decomposition of $X$, see [CM09a], Corollary 5.3 (ii).

Let $G$ be the closure of the projection of $\Gamma$ to $\operatorname{Isom}\left(Y_{1}\right) \times \cdots \times \operatorname{Isom}\left(Y_{q}\right)$. Then $G$ acts properly cocompactly on $Y=Y_{1} \times \cdots \times Y_{q}$. Therefore it contains an element $g$ acting as a hyperbolic isometry on $Y_{i}$ for all $i$ by Proposition 5. Since $\Gamma$ maps densely to $G$ and since the stabiliser of each point of $Y$ in $G$ is open by [CM09a], Theorem 1.2, it follows that $\Gamma$-orbits on $Y \times Y$ coincide with the $G$-orbits. In particular, given $y \in \operatorname{Min}(g)$, we can find $\gamma_{Y} \in \Gamma$ such that $\gamma_{Y}\left(y, g^{-1} y\right)=(g y, y)$. Since $\angle_{y}\left(\gamma_{Y}^{-1} y, \gamma_{Y} y\right)=\angle_{y}\left(g^{-1} y, g y\right)=\pi$, we infer that $\gamma_{Y}$ is hyperbolic and has an axis containing the segment $\left[g^{-1} y, g y\right]$. In particular $\gamma_{Y}$ acts as a hyperbolic isometry on $Y_{i}$ for all $i$.

Let $\gamma_{Y}=(\alpha, h)$ be the decomposition of $\gamma_{Y}$ along the splitting $\operatorname{Isom}(X)=$ $\operatorname{Isom}(M) \times \operatorname{Isom}(Y)$. By construction $h$ acts as a hyperbolic isometry on $Y_{i}$ for all $i$.

Let $U \leq \operatorname{Isom}(Y)$ be the pointwise stabiliser of a ball containing $y, \gamma_{Y} y$ and $\gamma_{Y}^{-1} y$. Notice that every element of $\operatorname{Isom}(Y)$ contained in the coset $U h$ maps $y$ to $h y$ and $h^{-1} y$ to $y$, and therefore acts also as a hyperbolic isometry on $Y_{i}$ for all $i$.

On the other hand $U$ is a compact open subgroup of Isom $(Y)$ by [CM09a], Theorem 1.2. Set $\Gamma_{U}=\Gamma \cap(\operatorname{Isom}(M) \times U)$. Notice that $\Gamma_{U}$ acts properly and cocompactly on $M$ by [CM09b], Lemma 3.2. In other words the projection of $\Gamma_{U}$ to $\operatorname{Isom}(M)$ is a cocompact lattice. Abusing notation slightly, we shall denote this projection equally by $\Gamma_{U}$.

By the appendix from [BL93] (see also [Pra94] for an alternative argument), the group $\Gamma_{U}$ contains an element $\gamma_{M}$ acting as an $\mathbb{R}$-regular element on $M$. By [PR72], Lemma 3.5, there is a Zariski open set $V=V\left(\gamma_{M}\right)$ in $\operatorname{Isom}(M)$ with the following property. For any $\delta \in V$ there exists $n_{\delta}$ such that an element $\gamma_{M}^{n} \delta$ is $\mathbb{R}$-regular for any $n \geq n_{\delta}$. By the Borel density theorem, the intersection $\Gamma_{U} \cap V \alpha^{-1}$ is nonempty. Pick an element $\delta \in \Gamma_{U} \cap V \alpha^{-1}$. Then $\delta \alpha \in V$ which means by definition that $\gamma_{M}^{n} \delta \alpha$ is $\mathbb{R}$-regular for all $n \geq n_{0}$ for some integer $n_{0}$.

Pick an element $\gamma_{M}^{\prime} \in \Gamma$ (resp. $\delta^{\prime} \in \Gamma$ ) which lifts $\gamma_{M}$ (resp. $\delta$ ). Set

$$
\gamma=\left(\gamma_{M}^{\prime}\right)^{n_{0}} \delta^{\prime} \gamma_{Y} \in \Gamma_{U}
$$


The projection of $\gamma$ to $\operatorname{Isom}(M)$ is $\gamma_{M}^{n_{0}} \delta \alpha$ and is thus $\mathbb{R}$-regular. The projection of $\gamma$ to Isom $(Y)$ belongs to the coset $U h$, and therefore acts as a hyperbolic isometry on $Y_{i}$ for all $i$.

\section{References}

[BS91] V. Bangert and V. Schroeder, Existence of flat tori in analytic manifolds of nonpositive curvature. Ann. Sci. École Norm. Sup. (4) 24 (1991), 605-634. Zbl 0760.53032 MR 1132759

[BL93] Y. Benoist and F. Labourie, Sur les difféomorphismes d'Anosov affines à feuilletages stable et instable différentiables. Invent. Math. 111 (1993), 285-308. Zbl 0777.58029 MR 1198811

[BH99] M. R. Bridson and A. Haefliger, Metric spaces of non-positive curvature. Grundlehren Math. Wiss. 319, Springer-Verlag, Berlin 1999. Zbl 0988.53001 MR 1744486

[CM09a] P.-E. Caprace and N. Monod, Isometry groups of non-positively curved spaces: structure theory. J. Topol. 2 (2009), 661-700. Zbl 1209.53060 MR 2574740

[CM09b] P.-E. Caprace and N. Monod, Isometry groups of non-positively curved spaces: discrete subgroups. J. Topol. 2 (2009), 701-746. Zbl 1187.53037 MR 2574741

[CS11] P.-E. Caprace and M. Sageev, Rank rigidity for CAT(0) cube complexes. Geom. Funct. Anal. 21 (2011), 851-891. Zbl 1266.20054 MR 2827012

[Gro93] M. Gromov, Geometric group theory (Sussex, 1991), vol. 2: Asymptotic invariants of infinite groups. London Math. Soc. Lecture Note Ser. 182, Cambridge University Press, Cambridge 1993. Zbl 0841.20039 MR 1253544

[Kle99] B. Kleiner, The local structure of length spaces with curvature bounded above. Math. Z. 231 (1999), 409-456. Zbl 0940.53024 MR 1704987

[Pra94] G. Prasad, R-regular elements in Zariski-dense subgroups. Quart. J. Math. Oxford Ser. (2) 45 (1994), 541-545. Zbl 0828.22010 MR 1315463

[PR72] G. Prasad and M. S. Raghunathan, Cartan subgroups and lattices in semi-simple groups. Ann. of Math. (2) 96 (1972), 296-317. Zbl 0245.22013 MR 0302822

[Rua01] K. E. Ruane, Dynamics of the action of a CAT(0) group on the boundary. Geom. Dedicata 84 (2001), 81-99. Zbl 0984.20027 MR 1825346

[Sel60] A. Selberg, On discontinuous groups in higher-dimensional symmetric spaces. In Contributions to function theory (Internat. Colloq. Function Theory, Bombay, 1960), Tata Institute of Fundamental Research, Bombay 1960, 147-164. Zbl 0201.36603 MR 0130324

[Swe99] E. L. Swenson, A cut point theorem for CAT(0) groups. J. Differential Geom. 53 (1999), 327-358. Zbl 1038.20029 MR 1802725

Received December 21, 2011; revised January 28, 2012 
P.-E. Caprace, Université catholique de Louvain, IRMP, Chemin du Cyclotron 2, 1348 Louvain-la-Neuve, Belgium

E-mail: pe.caprace@uclouvain.be

G. Zadnik, Inštitut za matematiko, fiziko in mehaniko, Jadranska ulica 19, SI-1111 Ljubljana, Slovenia

E-mail: zadnik@fmf.uni-lj.si 